\title{
Double lung transplantation in patients with lung atelectasis and complete mediastinal shift
}

\author{
Daniel Neto ${ }^{1}$, Ernest Chan ${ }^{1}$, and Pablo Sanchez ${ }^{2}$ \\ ${ }^{1}$ University of Pittsburgh Medical Center \\ ${ }^{2}$ University of Pittsburgh Medical Center Health System
}

July 2, 2021

\begin{abstract}
Mediastinal shift and chest wall retraction limits the indication of a double lung transplant in patients with complete destruction of lung parenchyma. We report outcomes as well as preoperative and operative management in two patients with significant lung volume reduction and mediastinal shift due to bronchiectasis of the right lung.
\end{abstract}

Double lung transplantation in patients with lung atelectasis and complete mediastinal shift Daniel M Neto MD ${ }^{1}$, Ernest G. Chan MD MPH${ }^{1}$, Pablo G. Sanchez MD PhD ${ }^{1}$

1 Division of Thoracic Surgery, Department of Cardiothoracic Surgery, University of Pittsburgh Medical Center, Pittsburgh PA

Funding Source: None

Conflict of Interest: None

Institutional Review Board: The University of Pittsburgh Review Board approved retrospective review of these cases

Patient Consent: not applicable

Permission to reproduce material from other sources: not applicable

Clinical trial registration: not applicable

Word Count: 1260

Corresponding Author:

Pablo Sanchez, MD, PhD

Surgical Director of Lung Transplant and ECMO

Department of Cardiothoracic Surgery

University of Pittsburgh

sanchezpg@upmc.edu

Phone: 412-648-6315

\section{ABSTRACT}


Mediastinal shift and chest wall retraction limits the indication of a double lung transplant in patients with complete destruction of lung parenchyma. We report outcomes as well as preoperative and operative management in two patients with significant lung volume reduction and mediastinal shift due to bronchiectasis of the right lung.

\section{Key Clinical Message}

For patients with complete atelectasis of one lung and severe mediastinal shift double lung transplant without the need for lung reduction is a feasible option providing these patients with the maximum benefit of lung volumes after transplant.

\section{INTRODUCTION}

Bronchiectasis is a heterogeneous disease featured by irreversible airway dilatation with chronic bronchial infection and inflammation (1). The conditions that directly affect airway clearance, such as ciliary dyskinesia, obstruction or cystic fibrosis, can lead to accumulation of secretions, causing what is called the "vicious cycle hypothesis" (2). The accumulation of secretions leads to infection, bronchial dilatation, resulting in more accumulation, closing the cycle. $(2,3)$ In rare settings, this constant cycle can ultimately create a retracted and atelectatic lung resulting in a mediastinal shift. This can have technical, clinical, and physiologic implications especially in patients with end-stage lung disease requiring lung transplantation. We report two uncommon patients presenting complete atelectasis of right lung with a prominent shift of the mediastinum to the right side who underwent successful sequential double lung transplantation.

\section{CASE REPORT}

\section{Patient 1}

A 58year-old female with Kartagener's syndrome presented with recurrent hospitalizations for pneumonia/bronchitis. She had a significant decrease in her functional status leading up to her transplant, requiring 2 to $4 \mathrm{~L}$ of oxygen at rest and upwards of $6 \mathrm{~L}$ with exertion. Due to the recurrent infectious exacerbations, the right lung collapsed, resulting in a dramatic shift in the mediastinum to the right side (Figure 1A ). The patient underwent sequential double lung transplant on cardiopulmonary bypass. Due to allograft size mismatch, the patient required a delayed chest closure on postoperative day (POD) 2 in the operating room (OR) after a chest washout with antibiotics. No donor lung volume reduction was necessary to close her chest. She did not require any postoperative cardiopulmonary or support. She was extubated on POD 6 and was discharged from the hospital on POD 38. Both lungs were fully expanded, and the mediastinum was centered. (Figure 1B).

\section{Patient 2}

A 35year-old male with cystic fibrosis (CF) had a recurrent history of hemoptysis as well as infectious exacerbations in the past. Due to these exacerbations, the patient developed a retracted right lung with concurrent right sided mediastinal shift (Figure 2A ). He underwent a sequential double lung transplantation on cardiopulmonary bypass support. Due to intraoperative coagulopathy and size mismatch, we elected to perform a delayed closure on POD 2 in the OR after a chest washout with antibiotics. No donor lung volume reduction was necessary to close his chest. His hospital course was complicated with respiratory insufficiency requiring tracheostomy and acute kidney injury requiring renal replacement therapy. Prior to being discharged on POD 52, his tracheostomy was decannulated and his kidney function returned to normal levels. Both lungs were fully expanded, and the mediastinum was centered. (Figure 2B )

\section{COMMENT}

Bronchiectasis, regardless of etiology, can result in a significant decrease in quality of life. While surgical treatments are not the first line of treatment, it is therefore important to relegate these treatment options for those that would benefit in quality of life due to relief from symptoms such as chronic cough, excessive sputum production and hemoptysis, when medical therapies fail. Among all surgical forms for bronchiectasis treatment, lung resection (sublobar and lobar) of focal compromised areas is the most frequent and acceptable 
possibility. Resection of the compromised segments may reduce the bouts of infection. This results in a significant improvement in the patient quality of life and low morbidity and mortality after surgery (4). Improvements in surgical technique and use of effective antibiotics led to this dramatic change in surgical results.

Lung transplantation is another viable surgical option for selected patients with advanced bronchiectasis of any origin (CF's and non-CF's), who have poor quality of life and are at risk of death once the decrease on their lung capacity compromises their daily activities or leads to recurrent hospital admissions. Cystic fibrosis encompasses approximately $26 \%$ of all total bilateral lung transplantations while non-CF bronchiectasis patients accounts for $4.5 \%$ of all bilateral lung transplantations (5). Despite the challenges that CF patients present, the overall survival after lung transplantation is more favorable than that seen in patients with chronic obstructive pulmonary disease or pulmonary fibrosis.

In rare, advanced cases patients may present with a completely collapsed ipsilateral lung with concurrent retracted asymmetric hemithorax. This often poses significant surgical challenges in lung transplantation and several surgical techniques have been presented in several small case reports with good results. Jougon et al. reported long term outcomes in a case report of a patient undergoing a left lung transplantation with a concurrent right pneumonectomy in a patient who developed right sided bronchiectasis. They expressed concern with regards to an extensively retracted right hemithorax that would have compromised respiratory mechanics and therefore allograft function if a normal sized right lung was implanted without any major volume reduction (6). Other groups would also describe the combination of an unilateral lung transplant after a staged pneumonectomy. Samano and colleagues presented a case series of two patients who underwent bilateral sequential lung transplantation in the setting of bronchiectasis and asymmetric thoraces after undergoing lower lobectomies. In their series, due to the difference in size of the retracted hemithoraces, the surgical group elected to perform lower lobectomies of the donor lungs at the time of transplantation. This allowed for chest closure without any cardiopulmonary compromise (7).

In the current report, because of the extensive mediastinal shift resulting from their disease process, three possible surgical approaches were discussed at our lung transplant multidisciplinary meeting. Unilateral lung transplantation with contralateral pneumonectomy, left lung transplant plus right lobar transplant or sequential double lung transplantation. Unilateral lung transplantation was not considered as an option because the risk of allograft contamination via the non-transplanted native lung is high (8). Unilateral lung transplantation with contralateral pneumonectomy was deemed a feasible technique and has been previously described as well. In this situation, the pneumonectomy could be done in a staged fashion or at the time of unilateral transplantation $(9,10)$.

Ultimately, as described, we elected to perform bilateral sequential lung transplantation despite the reduced size of the right chest cavity. Although the mediastinal shift required significant dissection of the pericardium and the atelectatic lung in the operating room at the time of transplantation, we found that the accommodation of the donor lungs and the return of the mediastinum to a more anatomical situation happened early after the surgery without significant hemodynamic compromise. This response shows that the sequential double lung transplantation is thoroughly possible, even in non-optimal anatomical conditions. Furthermore, this procedure leads to an increased post-transplantation pulmonary capacity.

In order to accomplish these transplants, three surgical aspects are worth mentioning. First, we elected to leave the chest open after successful implantation due to size mismatch especially in the setting of a shifted mediastinum. This allowed for the allografts as well as the chest cavities to accommodate to one another, noting a return to normal anatomy on the first POD1 chest x-ray. The second aspect is the use of antibiotic solution during the chest washout and closure. These patients are frequently colonized by many different species of bacteria and it is common to develop an empyema after lung transplantation. Therefore, chest washouts with antibiotics may prevent this complication, and we have used this procedure in all of our patients with such characteristics. The third aspect is the use of antibiotics targeting those species of bacteria or fungus previously cultured in the sputum in the pre-operative evaluation. In our practice this is a sine qua non condition after lung transplantation. 
In conclusion, double lung transplantation is a safe and feasible option in patients with bronchiectasis with concurrent mediastinal shift. Favorable outcomes seen after sequential double lung transplantation in these patients were achieved by the used of delayed chest closure with concurrent antibiotic driven thoracic cavity washout.

\section{Figure Legends}

Figure 1: Case 1: Preoperative CT scan (A) showing right lung bronchiectasis and atelectasis resulting in mediastinal shift to the right. Postoperative CT scan (B) showing adequate right lung expansion after bilateral sequential lung transplantation and medialization of the mediastinal structures.

Figure 2 : Case 2: Preoperative CT scan (A) showing extensive right lung bronchiectasis and atelectasis resulting in an even more severe mediastinal shift to the right. Postoperative CT scan (B) showing adequate right lung expansion after bilateral sequential lung transplantation and medialization of the mediastinal structures

\section{REFERENCES}

1. King P, Holdsworth S, Freezer N, Holmes P. Bronchiectasis. Int Med J 2006;36:729-737.

2. Feldman C. Bronchiectasis: new approach to diagnosis and management. Clin Chest Med 2011;32:535546.

3. Pasteur MC, Bilton D, Hill AT. British Thoracic Society guideline for non-CF bronchiectasis. Thorax 2010;65:1-58.

4. Fan LC, Liang S, Lu HW, Fei K, Xu JF. Efficiency and safety of surgical intervention to patients with non-cystic fibrosis bronchiectasis: a meta-analysis. Sci Rep 2015;5:17382.

5. Hayes D, Meyer K. Lung transplantation for advanced bronchiectasis.Sem Resp Crit Care Med 2010;31:123-138.

6. Jougon J, Dromer C, Mac Bride T, Velly JF. Synchronous left lung transplantation and right pneumonectomy for end-stage bronchiectasis through clamshell approach. Specific problems. Eur J Cardiothorac Surg 2002;22(5):833-835.

7. Samano MN, Waisberg DR, Villiger LE, Pego-Fernandes PM, Jatene FB. Bilateral lung transplantation in asymmetric thorax: Case reports. Transplant Proc 2008;40(3):872-874.

8. Soulimas R, Mostafa A, Guillemain R, Boussaud V, Amrein C, Chevalier P. Single-lung transplantation for cystic fibrosis and metachronus pneumonectomy: case reports. Transp Proc 2008;40:3594-3595.

9. Piotrowski JA, Splittgerber FH, Donovan TJ, Ratjen F, Zerkowski HR, Single-lung transplantation in a patient with cystic fibrosis and an asymmetric thorax. Ann Thorac Surg 1997:64:1456-1459.

10. Forty J, Hasan A, Gould FK, Corris PA, Dark JH. Single lung transplantation with simultaneous contralateral pneumonectomy for cystic fibrosis. J Heart Lung Transplant 1994;13:727-730.

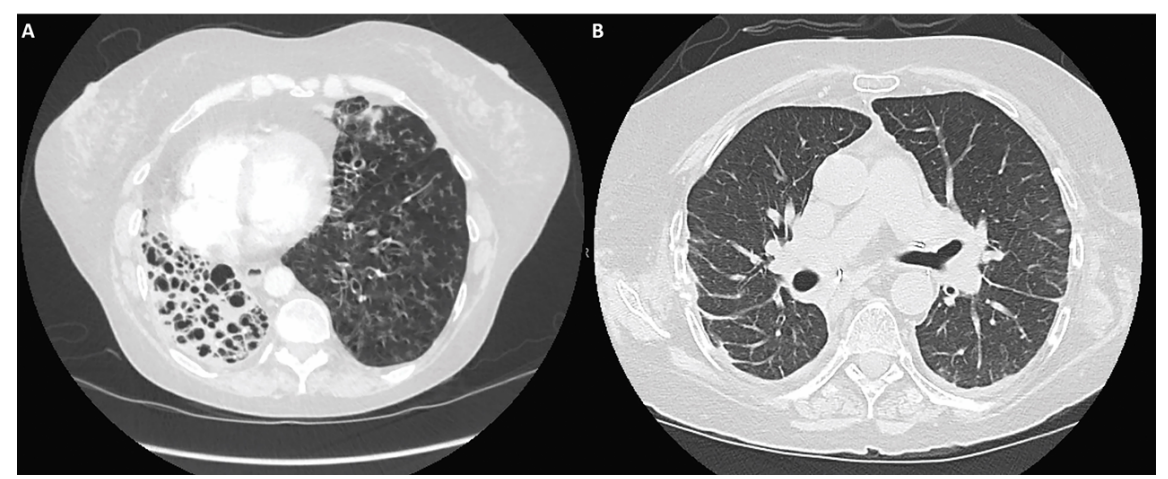




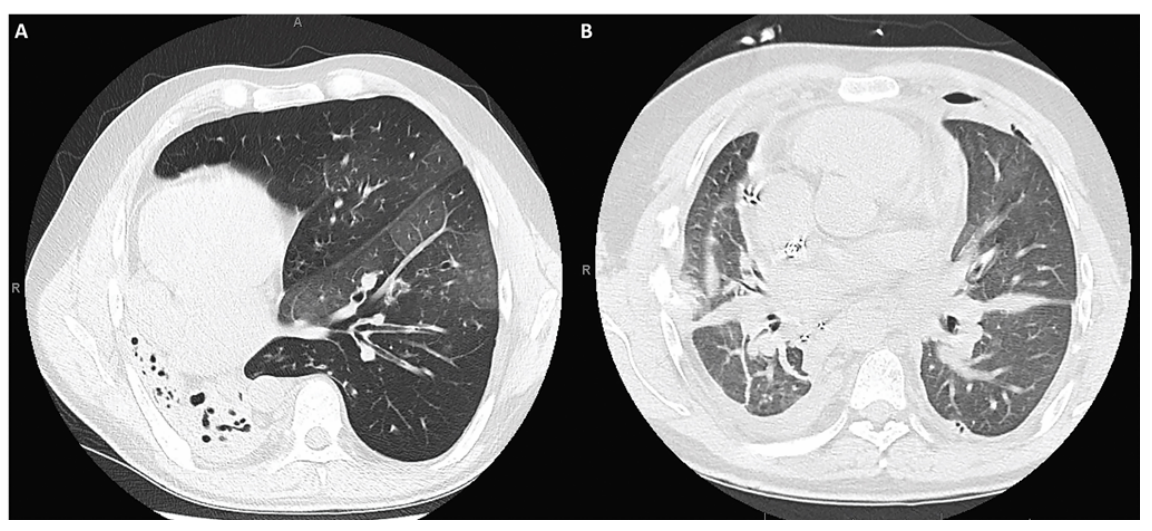

